\title{
Analysis of Key Technologies of Bridge Damage Detection Based on Visual Recognition
}

\author{
Tan Xixue ${ }^{1}$, Zhang Yunkai ${ }^{1}$, Li Guohua ${ }^{1 *}$, Pei Xuewen ${ }^{2}$ \\ ${ }^{1}$ School of civil and transportation engineering Beijing University of Civil Engineering and Architecture, Beijing 102616 China \\ ${ }^{2}$ Beijing-Dublin International College at BJUT, Beijing 100124 China
}

\begin{abstract}
In the identification of bridge damage in the series of deflection-affecting lines, noise signals due to axle coupling often occur. Removing these noise signals has become a key technique for effectively identifying damage. Taking the main line bridge of a overpass project in Fuzhou City of Fujian Province as an example, we collected the deflection a data of the bridge by using HPON-X target-free bridge deflectometer and used YOLOv3 algorithm for deep learning of the vehicle load position. The data were measured and studied by using DB9 wavelet de-noising method. The research shows that this method can greatly reduce the influence of vehicle bridge interaction on deflection influence line, and can enhance the accuracy and speed of bridge damage detection.
\end{abstract}

\section{Introduction}

Bridge is an important part of transportation engineering, in the development of national economy, promote cultural exchanges and consolidate national defense and other plays an irreplaceable role, the structural security of bridges is the primary prerequisite for its role. Therefore, it is of great theoretical significance and engineering value to study the method of rapid and accurate identification of bridge structural damage. In bridge damage recognition, signal noise cancellation for detection data becomes the key technology to determine whether damage can be effectively identified.

\section{Analysis of vehicle bridge interaction}

From the perspective of vehicle dynamics, automobile or railway vehicle is mainly composed of car body, bogie, wheel and spring suspension device. When the vehicle or train is driving on the bridge structure, the bridge structure not only bears the static force, but also the moving load, as well as the vibration inertia force of the bridge and vehicle. The running vehicles will have a dynamic impact on the bridge structure, causing the bridge to vibrate, and the vibration of the bridge will in turn affect the vehicles driving on the bridge ${ }^{[1]}$. Vehicle and Bridge constitute a multi-degree-of freedom vibration system in which vibrations influence and interact with each other. This is the vehicle bridge interaction.

There are many influencing factors of the vehiclebridge interaction effect, and the reasons for the vibration of the vehicle-bridge system are analyzed below. The engine of the vehicle and the power unit of the locomotive themselves will produce certain vibrations, which will cause the vibration of the system. When a vehicle or train passes a bridge at a certain speed, the unevenness between the road, line and wheels will cause the internal self-excited vibration of the system to form a forced vibration of the system.

The Italian scholar Diana has conducted an in-depth research on the problem of vehicle-bridge dynamic interaction. He comprehensively analyzed the discrete methods of different forms of vehicles and bridges, and established 23 free centralized mass vehicle models. And he carried out the theoretical analysis and actual measurement comparison on a steel truss railway bridge on the Milan-Rome railway line (as shown in Figure1)

\footnotetext{
* Corresponding author's e-mail: liguohua@bucea.edu.cn
} 


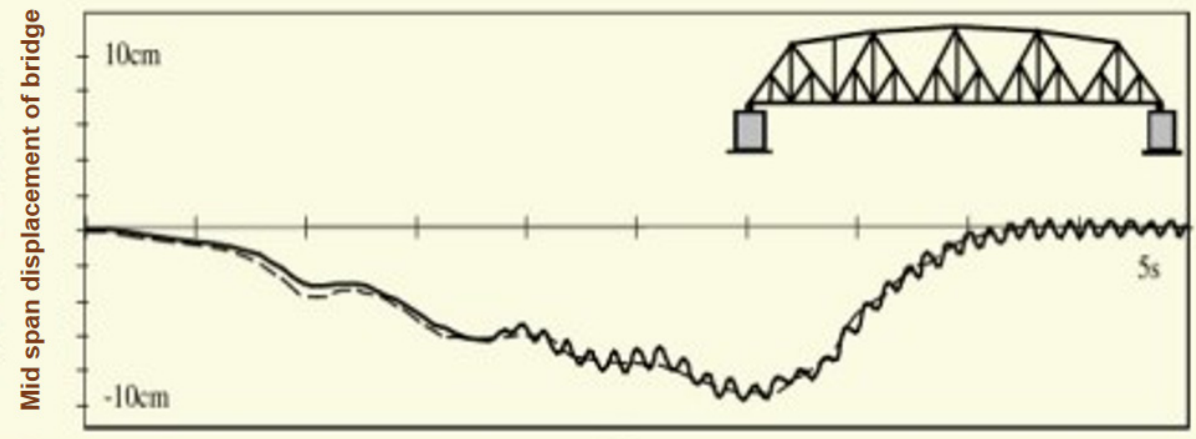

Figure 1 Mid span displacement of Milan-Rome railway bridge

By comparing the theoretically analyzed mid-span displacement with the measured bridge mid-span displacement, it is found that the measured displacement will be affected by the vehicle bridge interaction effect, showing a fluctuating curve. Therefore, in the damage identification method of continuous beam bridge structure based on visual tracking technology, the vehicle bridge interaction effect will have an impact on the collected deflection values.

With the development of computer technology, the research on the vehicle bridge interaction is more accurate. The single bridge model is used to analyze the simply supported beam and continuous beam vehicle bridge interaction. The plate shell, solid or plate shell combing solid finite element template beam bridge is used in the complex vehicle bridge interaction vibration research ${ }^{[2-5]}$.

The simulation of vehicle load also makes different choices according to different situations. When the vehicle mass is small and the vibration of the vehicle has little influence on the bridge, the moving load can be used to simulate the action of the vehicle driving, which is widely used in continuous beam and plane curved bridges. However, when the vehicle mass is large or will have a great impact on the bridge, the simulation error of the moving load model is large, so it is necessary to consider the vehicle as a dynamic rigid body, and consider its inertia problem in depth.

\subsection{Data filtering and noise reduction}

The deflection signal collected by the sensor will be mixed with interference components, which are the socalled noise. When the noise generated by other factors is too large, the useful original signal is not prominent, so it is difficult to collect useful information and use it. Technically, $\mathrm{S} / \mathrm{N}$ is defined as signal-to-noise ratio, which is used to measure the proportional relationship between signal and noise.

The commonly used filtering functions for noise reduction include Hear wavelet, bioNr.Nd wavelet system, Daubechies (dbN) wavelet system, Morlet (morl) wavelet, Symlet (symN) wavelet system, DOG (Difference of Gaussian) wavelet and Meyer function.

The wavelet function uses simple harmonic function as the base function to approximate any signal, but it is a series of functions with variable scales, which makes the wavelet function have good time-frequency positioning characteristics and adaptive ability to signal. The filtering effect of different wavelets is different, and the effect of wavelet denoising is affected by two factors: 1. which wavelet generating function is used for wavelet transformation and signal reconstruction; 2. which threshold selection and readjustment method is used to process wavelet coefficients ${ }^{[6]}$.

Lv Ruilan compared the noise reduction effect of each wavelet through spectral data and found that Daubechies9 (i.e. db9 wavelet) and Symlet7, 11, 14, 15 wavelet can achieve the best performance of noise reduction.

Daubechies wavelet function is proposed by Inrid Daubechies, a famous European wavelet analysis researcher ${ }^{[7]}$.Hypothesi $P(y)=\sum_{k=0}^{N-1} C_{k}^{N-1+k} y^{k}$, in which, $C_{k}^{N-1+k}$ is the coefficient of the binomial, can be expressed as:

$$
\begin{aligned}
\left|m_{0}(\omega)\right|^{2} & =\left(\cos ^{2} \frac{w}{2}\right)^{N} P\left(\sin ^{2} \frac{w}{2}\right) \\
m_{0}(\omega) & =\frac{1}{\sqrt{2}} \sum_{k=0}^{2 N-1} h_{K} e^{-i k w}
\end{aligned}
$$

The following paper uses $\mathrm{db} 9$ wavelet to denoise the measured data for two reasons: first, the predecessors have used $\mathrm{db} 9$ wavelet transform to perform similar denoising processing; secondly, db9 wavelet has the best effect in the noise reduction performance experiment.

\subsection{Real bridge analysis}

Since it is not possible to go out for real bridge detection during the epidemic, we select the measured deflection data of a main line bridge of an overpass project in Fuzhou City, Fujian Province for research.

The overpass is a prestressed concrete three-span continuous bridge. The measuring points are set in the middle of the side span. The HPQN-X target-free bridge deflection meter is used to measure the deflection. The dynamic test adopts a $50 \mathrm{~mm}$ lens and the sampling frequency is $117 \mathrm{hz}$. 


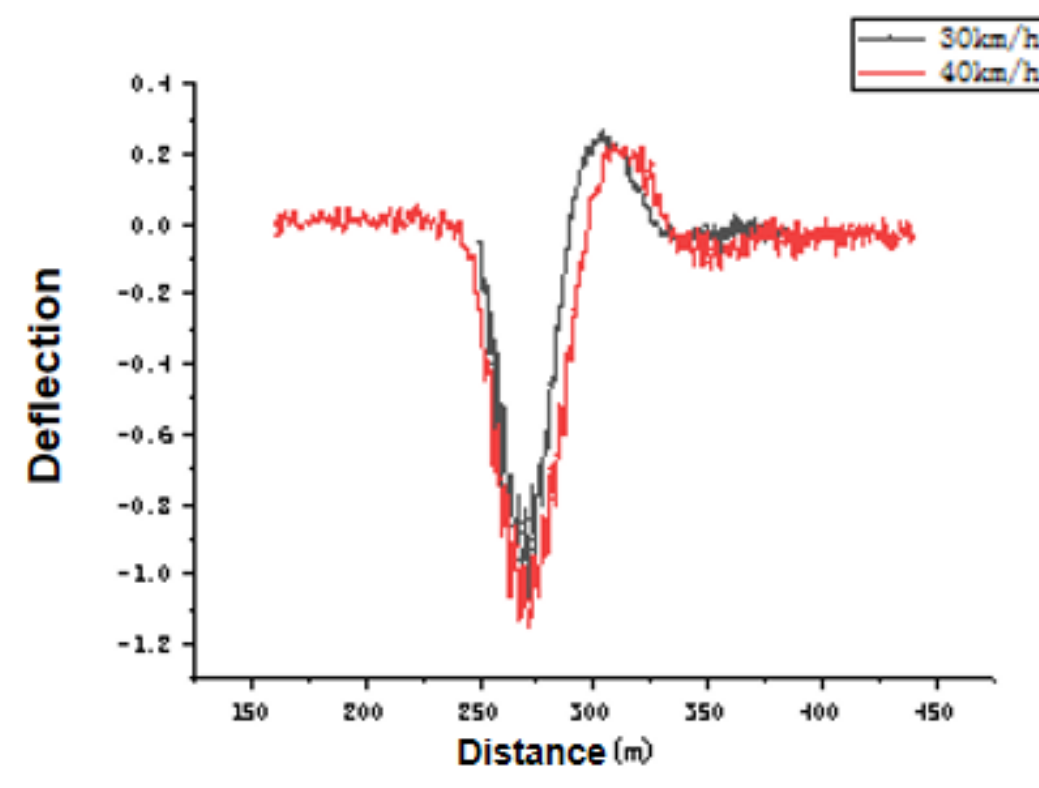

Figure 2 Image of dynamic load deflection influence line under different speeds

The bridge span is $30 \mathrm{~m}+30 \mathrm{~m}+30 \mathrm{~m}$. Firstly, the measured influence lines of bridge side span under different vehicle speeds are fitted to obtain the image of influence line of structural dynamic load deflection, as shown in Figure 2.

There are many burr-like fluctuations in the image, which will cause large errors in the solution of the deflection influence line difference. The cause of burr is largely due to the interference of vehicle bridge interaction vibration. In our proposed visual trackingbased damage identification method for continuous beam bridge structure, the first derivative of the structure deflection influence line is the best identification parameter. If the fluctuation interference caused by vehicle bridge interaction is not eliminated, the accuracy of damage identification will be greatly reduced.

DB9 wavelet transform is used to denoise the signal, and sqtwolog is chosen as the threshold, because sqtwolog can remove the white noise in the signal. In the wavelet transform domain, the energy of Gauss white noise is better concentrated in the case of low amplitude than in other cases ${ }^{[8]}$. The corresponding formula is as follows:

$$
\delta=r\left(2 \log _{2} N\right)^{\frac{1}{2} \frac{M A D\left(d_{j}\right)}{0.6745}}
$$




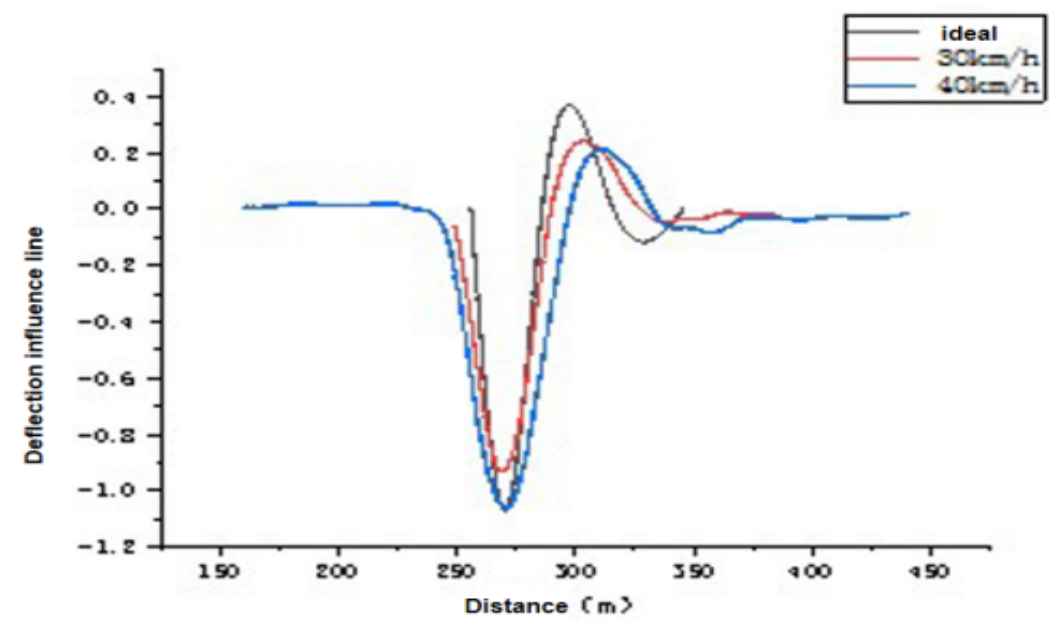

Figure 3 Dynamic load influence line after db9 wavelet analysis denoising

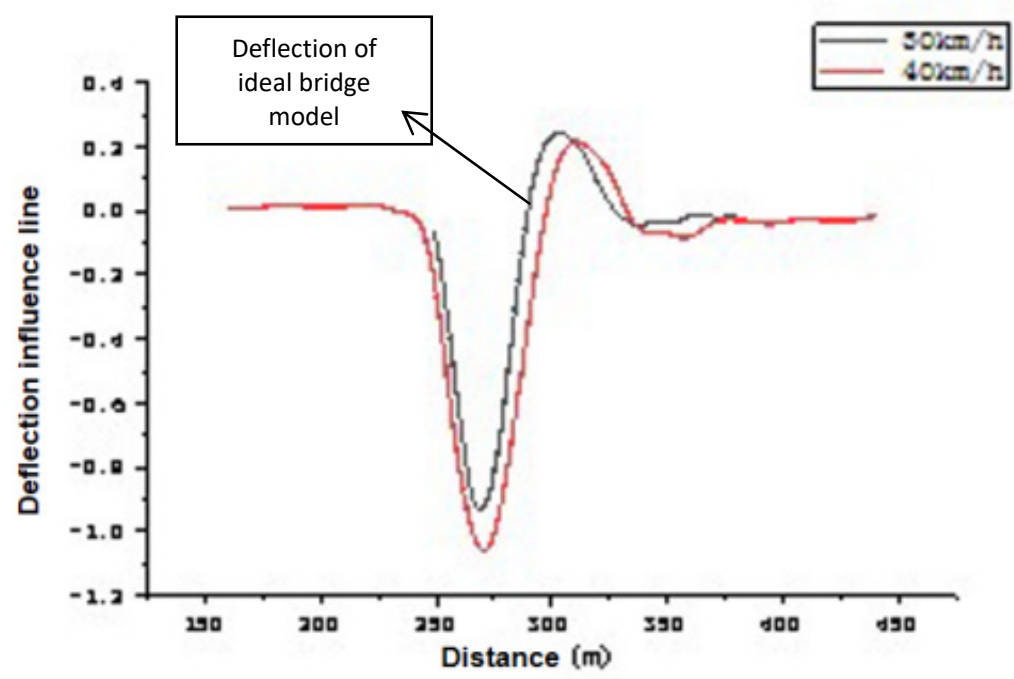

Figure 4 Dynamic load influence line and ideal measurement influence line after noise reduction

The dynamic influence line after processing is shown in Figure 3. Intuitively from the image, the burr of the curve has been eliminated, but the accuracy of the deflection influence line after wavelet transformation cannot be verified. Therefore, Midas civil is used to build a single beam model of the tested continuous beam bridge. The single-beam model considers the lateral distribution coefficient, so after the model is established, the lateral distribution coefficient is assigned according to the ratio between the image and the measured image, and the static theoretical influence line of the bridge is obtained. Put the theoretical static influence line and dynamic load influence line after DB9 wavelet analysis denoising into the same figure for comparison, as shown in Figure 4. It is found that the dynamic load influence line after noise reduction is highly coincident with the deflection influence line under static load, and the burrlike interference caused by the vehicle bridge interaction is eliminated, and the continuous beam bridge deflection influence line obtained under the visual tracking technology is greatly optimized.

\section{Application of visual tracking technology in bridge measurement}

\subsection{Technical support}

The so-called visual tracking refers to the detection, extraction, recognition and tracking of moving targets in the image sequence, so as to obtain the motion parameters such as the position and speed of the moving target, and then process and analyze the obtained data in depth to complete more advanced tasks ${ }^{[9]}$.

At present, it has been mainly used in traffic monitoring system for traffic statistics to ensure smooth traffic. Tai et al. ${ }^{[10]}$ developed a video surveillance system for traffic accident detection, which can automatically detect moving vehicles and determine their trajectories. VISATRAM (Vision System for Automatic Traffic Monitoring) system can monitor the behavior of vehicles in each lane to ensure smooth urban traffic. Haag and Nagel are devoted to the study of vehicle tracking of automatic driving. 
There is still a lot of room for the development of other research and application of visual tracking in the field of transportation, and the future research focus is how to achieve vehicle tracking quickly and accurately. The visual tracking technology used in this paper is based on deep learning.

Convolutional neural network based on deep learning has been integrated into people's life, and our target detection algorithm based on deep learning can recognize vehicle targets, so as to achieve the effect of accurate identification of load position. There are two kinds of target detection algorithms: single-stage and two-stage. The recognition accuracy of the single-stage algorithm is lower than that of the two-stage algorithm, but its recognition speed is much faster than that of the twostage algorithm. With the continuous optimization of the algorithm by scholars, the third version of the YOLO algorithm in the current single-stage algorithm. YOLOv3 has higher recognition accuracy and faster recognition speed. So this paper selects the YOLOv3 algorithm to identify vehicles.

The YOLOv3 algorithm was proposed by Joseph Redmon et al ${ }^{[11]}$, which uses a new network to perform feature extraction: Darknet-53 with 53 convolution layers. The convolution layer is the most important part of convolution neural network, the convolution kernel slides according to the parameters such as the step length, and slides once to make the inner product of the current position and the pixel at that position. The extraction of image features is determined by its convolution kernel, including the edges, corners, colors and so on of the image. As the convolution layers become deeper and deeper, the extracted features gradually transform from shallow geometric features to deep abstract features. Therefore, the YOLOv3 algorithm is as accurate as the SSD algorithm, but its speed is three times faster; The object classification uses Logistic instead of softmax to support multi label objects; The priori frame of nine scales can realize cross scale prediction, which can be applied to small target detection. The general technical route of applying this algorithm to vehicle recognition is shown in Figure 5.

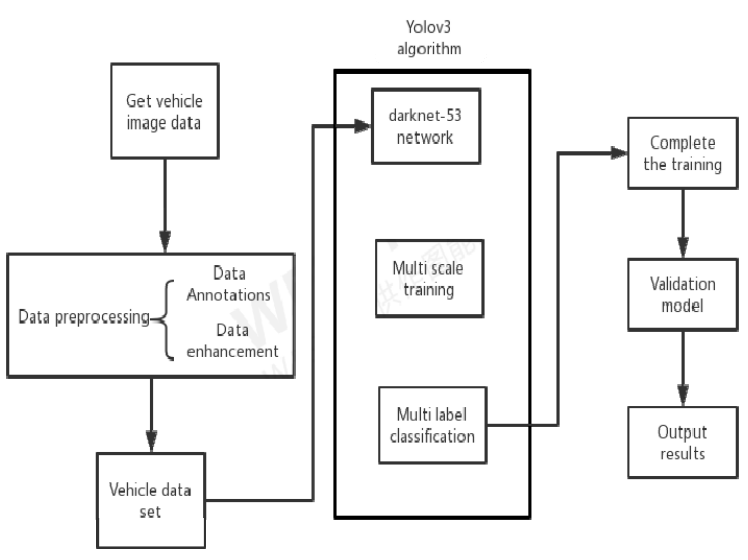

Figure 5 Technical route of visual tracking and recognition of vehicle based on yolov3 algorithm
Through the training of 3052 datasets, the computer can find the relationship and expression level between a large number of data, and finally achieve the effect of vehicle image recognition. Select a section of road recorded video and use the algorithm to identify it. The recognition effect is shown in Figure 6-9.

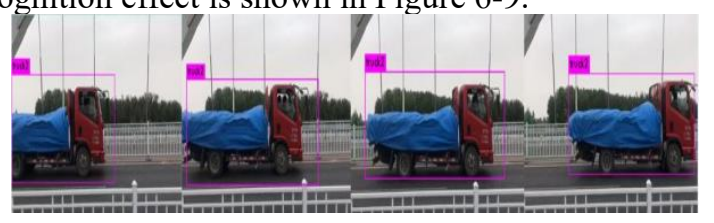

Figure 6 Recognition effect of two axle truck (truck 2)

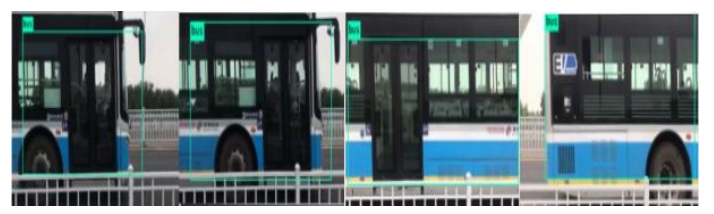

Figure 7 Bus recognition effect

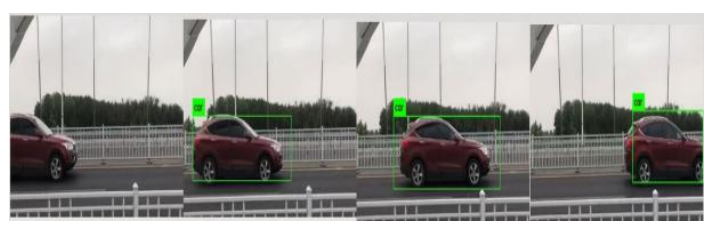

Figure 8 Recognition effect of cars

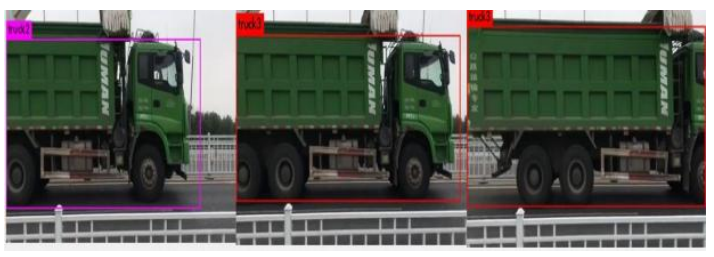

Figure 9 Recognition effect of three axle truck (truck 3)

In the video recognition, only when the car is not fully in the mirror and the three axle truck is not fully in the mirror, miss detection and false detection occur. Therefore, when the technology is applied to identify the vehicle load position in the real bridge detection, it should ensure that the vehicle is completely in the mirror and the camera instruments are arranged on both sides of the road to ensure that the vehicles will not miss the detection due to the overlap.

\subsection{Application method}

In the YOLOv3 algorithm, we can identify the vehicle load frame by frame through a piece of video to get the precise position of the vehicle load. The signal collected by the deflection sensor is denoised. The data after noise reduction corresponds one-to-one with the load acting position in each frame of the screen, and the deflection curve under the moving load is obtained, and the deflection influence line can be obtained after processing. Then apply the conclusion of Chapter 3-using the first derivative of the deflection influence line difference to locate the structural damage, the technical route map is shown in Figure 10. 


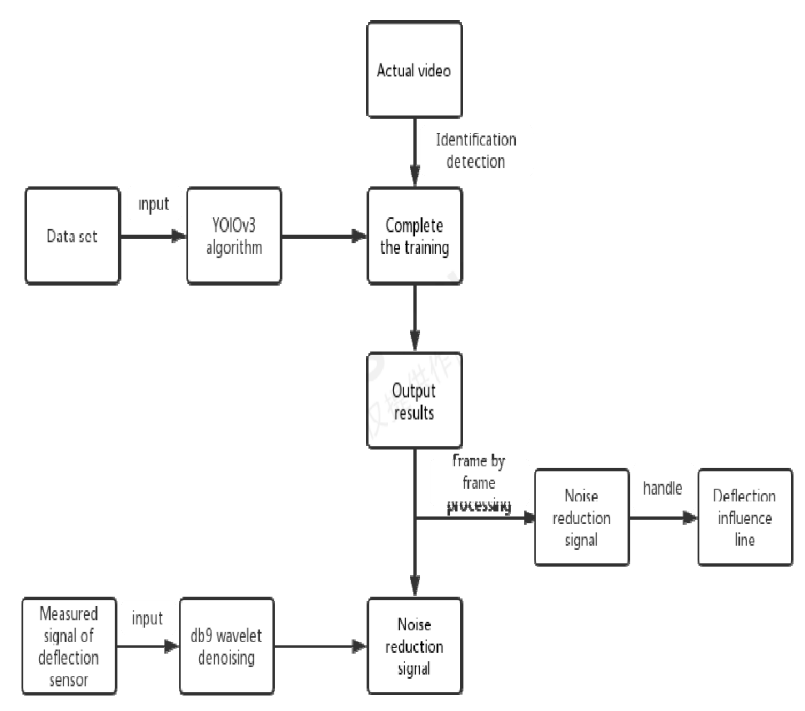

Figure 10 Technical route

\subsection{Application Value}

\subsubsection{Improvement of detection accuracy}

The traditional method to obtain the influence line of structural deflection under dynamic load is to keep the vehicle load at a uniform speed while driving on the bridge, and the deflection sensor collects the deflection data of the measuring points from the vehicle load entering into the bridge to leaving the bridge. The collected time-deflection data are processed. Taking the time when the load enters the bridge as the time origin, and then making the difference between the time corresponding to the deflection value and the time origin, the position deflection curve under the moving load can be obtained by multiplying the difference by the driving speed of the vehicle load on the structure, and then the influence line can be obtained from the position deflection curve.

Both the time-deflection data and the positiondeflection data obtained by the traditional method will be interfered by a variety of error factors. For example, in the acquisition of time data, the judgment of time origin relies on human vision to record the time when the vehicle is loaded on the bridge. The visual response time of human is generally $0.1-0.5 \mathrm{~s}$, so there is a certain time offset between the recorded time starting point and the actual value. In the acquisition of location data, the default vehicle always keeps the assumed speed. However, in the detection of real bridges, the load moving speed will not reach the ideal conditions due to the unevenness of the road surface, the driving technique of the driver and many other factors. Therefore, at the specified time node, the actual position of the load may be behind or ahead of the assumed position in data processing, and the specific error generated therein cannot be clearly quantified.

In the damage identification method of continuous beam structure based on visual tracking technology proposed in this paper, the computer is used to visually track the vehicle load, which can effectively eliminate the above-mentioned errors. Multiple cameras are placed on both sides of the bridge to record the movement of vehicle load on the bridge. Record the entire process of vehicle load driving on the bridge, and analyze the load video with a computer trained through the YOLOv3 algorithm. The computer can accurately extract the type of vehicle and the driving position information in each frame of the vehicle, without manual judgment and calculation of the load on the bridge time, so the visual reaction time error of the surveyor can be eliminated.

When the visual tracking technology identifies the vehicle position, there is no default vehicle driving at a constant speed. Instead it is based on the image to judge the position of the vehicle at every moment. Therefore, in the process of measurement, this method is not disturbed by the driver's technology and uneven speed caused by uneven road surface.

\subsubsection{Improvement of detection speed}

The traditional detection process requires uniform driving speed to ensure that the deflection influence line obtained after data processing is within a certain error range. Therefore, in order to achieve the measurement accuracy, it is necessary to block the traffic and drive evenly at different speeds, and take multiple sets of test data. However, blocking traffic for a long time will pay great economic value and time value.

The initial stage of structural damage identification detection using visual tracking technology is applied to short-term closed traffic, the traffic can be opened after the loaded vehicle passes by at a relatively uniform speed. The surveyors will carry out subsequent processing of the vehicle motion parameters in the video and the collected deflection parameters, so there is no need to require too much of the load moving speed. Therefore, 
the time of closing traffic is shorter than that of traditional methods.

In the next stage of structural damage identification and detection under data tracking technology, it can be done even without closing the traffic. At present, under the algorithm of YOLOv3, the computer can identify different types of vehicles, such as cars, buses, two axle trucks and three axle trucks. When the traffic volume of the bridge is small, camera equipment can be set up on both sides of the bridge, and mobile weighbridge can be set up at the entrance and exit of the bridge (as shown in Figure 11). The weight of all vehicles is known before driving into the bridge. The position of multiple vehicle loads on the bridge at the same time can be obtained by using visual tracking technology, so the size and position of the load on the bridge at each time can be determined. The deflection value of bridge measuring point under each known condition can be obtained by randomly detecting during the period of less traffic flow of bridge, and the deflection influence line of bridge structure can be obtained by processing it. Then use the conclusion of multi-point damage identification in Chapter 3 to locate the damage, which eliminates the interference of the structure damage identification process to normal traffic operation.

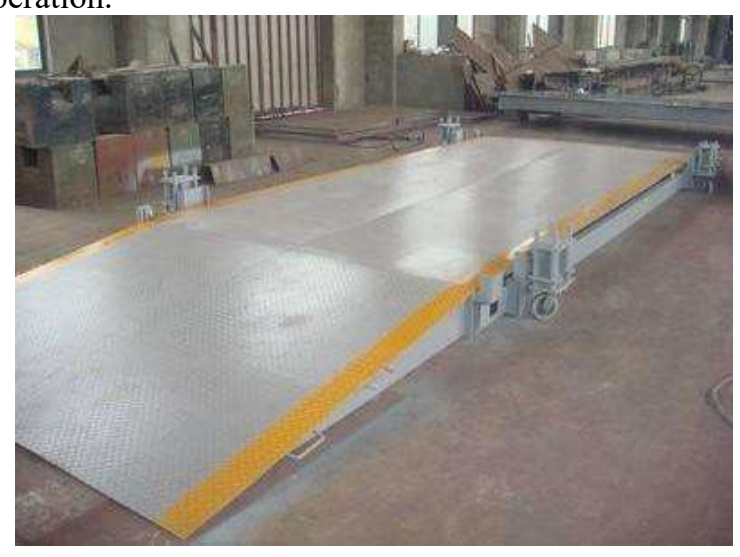

Figure 11 Mobile weighbridge

\section{Conclusions}

Taking the main line bridge of an overpass project in Fuzhou City of Fujian Province as an example, we collected the deflection data of the bridge by using HPQN-X target-free bridge deflectometer, and used YOLOv3 algorithm for deep learning of the vehicle load position, the data were measured and studied by using DB9 wavelet de-noising method, the research shows that this method can greatly reduce the influence of vehicle bridge interaction on deflection influence line, and can enhance the accuracy and speed of bridge damage detection. However, YOLOv3 algorithm cannot fully recognize the accurate position of the vehicle at present, your suggestion and correction is highly appreciated. In order to effectively identify the specific location of bridge damage and speed up detection, the study of similar algorithms has become particularly important. In future research, more emphasis should be placed on the optimization of algorithms so that they can more effectively meet modern detection needs.

\section{References}

1. An Yi. Research on Initial Deformation Limit Index for Long-span Passenger Dedicated Railway Ballastless Track Bridge[D]. Beijing Jiaotong University, 2009

2. Evaluation of impact factors for composite concretesteel cellular straight bridges. X. Zhang K. Sennah and J. B. Kennedy. Engineering Structures . 2003

3. Effect of Vehicle Velocity on the Dynamic Amplification of a Vehicle Crossing a Simply Supported Bridge[J] Sean P. Brady, Eugene J. O'Brien,Ale? ?nidari? Journal of Bridge Engineering . 2006 (2)

4. Experimental Evaluation of Dynamic Effects for a Selected Highway Bridge[J]Leslaw Kwasniewski, Jerry Wekezer, Garry Roufa, Hongyi Li,Jean Ducher, Jerzy Malachowski. Journal of Performance of Constructed Facilities. 2006 (3)

5. Finite element analysis of vehicle-bridge interaction[J]. Leslaw Kwasniewski, Hongyi Li, Jerry Wekezer, Jerzy Malachowski. Finite Elements in Analysis \& Design. 2006 (11)

6. Lv Ruilan, Wu Tiejun, Yu Ling. Performance Analysis of Threshold Denoising via Different Kinds of Mother Wavelets [J]. SPECTROSCOPY AND SPECTRAL ANALYSIS, 2004 (07): 826-829.

7. Long Dong. Tracking Key Technology Research of Video Audio Fusion Based on the Head of Robot[D]. Hebei University of Technology, 2007

8. He Xiaolei. A Research on Wavelet-based Speech Signal Denoising Algorithms and their Applications [D]. Guangxi Normal University, 2012.

9. Hou Zhiqiang, Han Chongzhao. A Survey of Visual Tracking[J]. Acta Automatica Sinica, 2006(04):603617.

10. Real-time multiple vehicle detection and tracking from a moving vehicle[J]. Margrit Betke, Esin Haritaoglu, Larry S. Davis. Machine Vision and Applications. 2000 (2)

11. Joseph Redmon, Ali Farhadi. YOLOv3: An Incremental Improvement [DB] arXiv: 1612. 08 